\section{Problem gambling - prevalence and mental health aspects in a psychiatric setting}

\section{J Chow-Fairhall', D Watkins' ${ }^{2}$, A de Castella ${ }^{2,3}$, J Kulkarni ${ }^{2,3}$}

'The Alfred Crisis Assessment \& Treatment/Psychiatric Triage Team; ${ }^{2}$ The Alfred Psychiatry Research Centre, The Alfred Hospital; and ${ }^{3}$ Monash University, Melbourne, Australia

Background: Suicidal behaviour is the most serious outcome of an activity that is promoted as recreation (Productivity Commission 1999). Understanding of the prevalence of problem gambling and its risk factors in a population accessing mental health services has important implications for clinicians in these settings.

Method: Over 6 months, consecutive presentations of people who were seen either in the community by The Alfred's Crisis Assessment and Treatment Team or who were admitted to the emergency department of The Alfred and seen by The Alfred's Psychiatry Triage were screened for suicidal ideas or intent and problem gambling. Positive cases were invited to participate in a study to investigate the prevalence of problem gambling, suicide and other mental health issues.

Results: Preliminary data indicate high levels of problem gambling in the sample. There are gender differences found in those with suicidal ideas or behaviours and problem gambling. Results on a range of other biopsychosocial measures are compared with other populations.

Conclusions: The results of the project highlight characteristics that can be contrasted with international data to broaden the understanding of mental health issues in people for whom gambling is a problem or is a problem for someone who is close to them, or that gambling is the reason behind their contact with a mental health service. Further, it underscores the importance of including the identification of problem gambling as a factor for risk in clinical mental health assessment and the need for further research.

\section{Obesity and metabolic syndrome in a psychiatric rehabilitation service}

\section{L-E Chua, S Tirupati}

Hunter New England Area Health Service, Centre for Brain and Mental Health Research, Newcastle, Australia

Background: The high prevalence of metabolic syndrome in people on antipsychotic medication is related to increased morbidity and mortality. It adversely influences management of chronic mental disorders in a rehabilitation setting in several ways, including poor treatment adherence and impediment to work and independent functioning.
Methods: A clinical audit of 216 patients with psychotic disorders in a rehabilitation unit was done to diagnose metabolic syndrome using the criteria International Diabetes Federation (2005).

Results: Overweight and obesity was identified in $89 \%$ and metabolic syndrome in $68 \%$ of the cohort. The risk for metabolic syndrome was 17 times more in obese people compared with normal-weight people. Polypharmacy was disproportionately associated with the syndrome. Case examples highlight that regular exercise, dietary control and GP consultation can reverse the adverse metabolic status without any change in antipsychotic drug regimen.

Conclusions: Management of metabolic syndrome should be an integral component of management of people on antipsychotic medication. A protocol for an active intervention program that needs to be initiated early and continued through the course of antipsychotic drug treatment is presented.

\section{Judged effectiveness of treatments for depression: a web-based survey}

\section{J Crawford, G Parker}

The Black Dog Institute, Sydney, Australia

Background: Previous research has indicated that many self-help and complementary treatments for depression are commonly used and rated highly in terms of their perceived helpfulness. The aim of the current study was to examine the frequency of use, and judged effectiveness, of a range of both professionally implemented strategies (including antidepressants and psychological therapies) and self-help strategies, by those who have ever experienced a depressive episode.

Methods: Individuals accessing the Web site of the Black Dog Institute (www.blackdoginstitute.org.au) were invited - if they had ever received treatment for an episode of depression - to participate in an anonymous online survey. A total of 2692 participants were asked whether they had ever received any of a set of different treatments for depression, including a range of antidepressants, psychological therapies, electroconvulsive therapy and more alternative strategies. Respondents rated the effectiveness of each strategy trailed.

Results: 'Effectiveness' scores were computed for each of 31 strategies (including 15 antidepressant medications) that had been trailed by at least 100 participants. The rank order of the 10 highest rating strategies was as follows: venlafaxine, exercise, cognitive behavioural therapy, 'other psychotherapy', IPT, escitalopram, citalopram, yoga/meditation, sertraline and counseling. Ratings identified differential perceived effectiveness of different antidepressants. 